\title{
Motivational Interviewing Tool for Health Recovery
}

\author{
Saurav $\operatorname{Kumar}^{1 *}$, Dr. Mona Srivastava ${ }^{2}$, Dr. Manushi Srivastava ${ }^{3}$, \\ Avadhesh Kumar ${ }^{4}$,Abhimanyu Gupta ${ }^{5}$
}

\section{ABSTRACT}

Motivational interviewing is a directive, client centered counseling style that aims to help client explore and resolve their ambivalence about behavior change. Motivational interviewing plays pivotal role in improvement of health condition although primarily it was developed for fighting with addiction problem but now it shows its contribution in clinical setting to promote weight reduction, dietary modification, exercise thus having a potential profound impact on heart disease, hypertension diabetes mellitus prompting to use safe sex practices and protecting from the risk of HIV and other STD diseases. Motivational interviewing stresses on way of communication from traditional advice giving to reflective listening. Principles of motivational interviewing are Express empathy, Avoid arguments, Develop discrepancies, Roll with resistance and Support Self efficacy. As, it has been seen that motivation plays key role in any person's life and many diseases flourishes due to our lack of motivation to change behavior and our life style that used to exacerbate morbidity. Motivational interviewing technique used to act as a catalyst to accelerate our activities through behavior change and protecting us from health related problems in future.

Keywords: Motivational interviewing, health, behavior change.

Behavior change in client is a major challenge for today's professional involved in medical management. Chronic diseases (e.g., cardiovascular diseases, mental health disorders, diabetes, and cancer) are flourishing the nation resulting in over-utilization of health care resources which in turn raises the costs and taxes the health care system as a whole.

\footnotetext{
${ }^{1}$ Research Scholar, Department of Psychiatry IMS ,BHU Varanasi, Uttar Pradesh, India

${ }^{2}$ Associate Professor, Department of Psychiatry IMS, BHU Varanasi, Uttar Pradesh, India

${ }^{3}$ Assistant Professor, Department of Community Medicine IMS,BHU Varanasi, Uttar Pradesh, India

${ }^{4}$ Research Scholar ,Department of Community Medicine IMS, BHU Varanasi, Uttar Pradesh, India

${ }^{5}$ Research Scholar ,Department of Community Medicine IMS, BHU Varanasi, Uttar Pradesh, India

*Responding Author

(C) 2016, S Kumar, M Srivastava, A Kumar, A Gupta; licensee IJIP. This is an Open Access Research distributed under the terms of the Creative Commons Attribution License (http://creativecommons.org/licenses/by/2.0), which permits unrestricted use, distribution, and reproduction in any Medium, provided the original work is properly cited.
} 


\section{Motivational Interviewing Tool for Health Recovery}

While our medical system spends much of our resources and energy on treating illness rather than cultivating health. The most galvanizing apparatus to achieve the desired result lies within the individual or the client. This is where a breed of interventional techniques come in and in this branch of patient centered techniques is the highly effective, behavior modifying approach known as motivational interviewing.

Motivational interviewing (MI), originally described by Miller in 1983 and more fully discussed in a seminal text by Miller and Rollnick in 1991, has been used extensively in the addiction field (Dunn, Deroo, \& Rivara, 2001; Noonan \& Moyers, 1997). There has been considerable recent interest on the part of public health, health psychology, and medical professionals in adapting MI to address other health behaviors and conditions, such as smoking, diet, physical activity, screening, sexual behavior, diabetes control, and medical adherence (Emmons \& Rollnick, 2001; Resnicow, DiIorio, et al., 2002), although primarily it was developed for manages relevance in prevention and management of substance use and various other disorder.

Miller and Rollnick (2002) defined MI as “a client-centered, directive method for enhancing intrinsic motivation to change by exploring and resolving ambivalence”. Motivational interviewing neither a discrete nor entirely new intervention paradigm but an amalgam of principles and techniques drawn from existing models of psychotherapy and behavior change theory. It can be thought of as an egalitarian concept that promotes equality among Practitioner and client during the session relationship is seen as partnership rather than an expert and recipient one. Motivational interviewing stresses on way of communication from traditional advice giving to reflective listening.

\section{MOTIVATIONAL INTERVIEWING PRINCIPLE AND TECHNIQUES}

Miller and Rollnick suggest the following clinical principles upon which MI is based: express empathy, develop discrepancy, avoid argumentation, roll with resistance, and support selfefficacy (Miller \& Rollnick, 1991)

Express Empathy: This principle calls for the practitioner to enter the patient's world. What are the patient's concerns? Why is change difficult? What outcomes does the patient want to see? What obstacles are in the way? What is the patient's background? In essence, the practitioner's ability to understand the patient's experience, and to communicate that understanding, what will help the patient achieve the desired change.

Develop discrepancy: This principle helps patients in understanding where they are and where they want to be. Patient present values and future goals a technique that acquaints patient about future scenario as a consequence of their decision making. For example, the practitioner may run through a list of positive and negative consequences of a decision to, say, continue to smoke or begin a cessation program. Part of the power in this approach lies in the patient's verbalizing the positive outcomes as weighed against the current course of behavior. 


\section{Motivational Interviewing Tool for Health Recovery}

Avoid argumentation: Any form of Conflicts and disagreement in a patient-practitioner relationship has no space in the process of motivational interviewing. In Contrast MI promotes freedom of open discussion with egalitarian approach and discourages any form of autocratic behavior from practitioner it is liberal and client has full freedom to express their feelings freely and any form of Coercion and force is suppressed.

Roll with résistance: As MI believes on collaborative approach any form of combative and deadly tone is discouraged and clients resistance is not encountered and challenged directly but instead flows with the resistance, moving toward a closer detection of clients perspective and mindset. The emphasis is given on clients view and counselor can provide their suggestions how to overcome obstacles in by their collaborative and joint effort.

Support Self efficacy: Developing belief inside the client about their potential and they can come out of problems by giving reference through their past effort that in past they have succeed and other ones who have been successful .They have potential hidden inside them they can swim across the pound of hindrance.

\section{Spirit of Motivational Interviewing are as follows:}

Collaboration: Counseling involves a partnership that respects and accepts the clients point of views. The counselor provides an atmosphere that is conducive rather than coercive to change.

Evocation: The potential and motivation for change are presumed to dwell within the client. Intrinsic motivation for change is enhanced by drawing on the client's own perceptions, goals, and values. Evocation is in turn compared with education, in which there is an assumption of a deficit in the client's "knowledge, insight, and/or skills" that must be corrected by the therapist.

Autonomy: The therapist respects the client's right and freedom for choice any form of pushing to act in particular way from therapist is discouraged.

Reflective Listening : The goals of reflecting back to the client include demonstrating empathy, affirming clients thoughts and feelings, and helping the client continue through the selfdiscovery process. Reflections involve several levels of complexity or depth, ranging from understanding content to exploring meaning and feeling (Carkhuff, 1993). Argumentation or direct persuasion is considered counterproductive and is to be avoided, as it is likely to produce defensiveness or resistance. Instead, the style is generally quiet and facilitative, and the relationship is more like a partnership or companionship than an expert/recipient one.

Resistance, on the other hand, is seen as a signal to change strategy. It is not opposed, but rather acknowledged and explored, with the view to shifting the patient's perceptions.

The techniques of MI are applied within the context of the ingredients for effective brief interventions, using the acronym FRAMES, namely Feedback, Responsibility for change lies within the individual, Advice giving, Menu of change options, Empathic style, and Self-efficacy is enhanced. In MI, however, advice is not given without the patient's permission, and when 


\section{Motivational Interviewing Tool for Health Recovery}

given, is accompanied by actively encouraging the patient to make his or her own choices (Miller, 1994).

MI do not proceed in a well manner when the practitioner argues that the patient has a problem and needs to change, and starts giving direct advice, or prescribes solutions to the problem without the patient's permission or without actively encouraging the patient to make their own choices. MI is also not being offered if the practitioner takes an authoritative/expert stance, leaving the patient in a passive role, or functions as a unidirectional information delivery system.

The client is encouraged to talk about their typical day, and thereby talk about their current behaviour in detail within a non-pathological framework. The client is asked to tell the positive and negative side of continuing same behavior. Some suggested questions are: "What are the good things about smoking and not so good things about smoking." Clients are encouraged to talk as much as or more than therapist. There is generally no direct attempt to annihilate denial, confront irrational or maladaptive beliefs, or convince or persuade. Instead, the goal is to help clients think about and verbally express their own reasons for and against change, how their current behavior or health status affects their ability to achieve their life goals or live out their core values.

To achieve these ends Motivational interviewing therapist have faith on reflective listening and positive affirmations rather than on direct questioning, persuasion, or advice giving clients are encouraged to think about their current satisfaction with life and what the future looks like both if they continue as they are and if they change their behavior. The client is invited to weigh up the pros and cons of changing his or her behavior.

\section{Similarities and Dissimilarities with other theoretical models}

MI is rooted in Rogers's person-centered approach to psychotherapy. Similar to Rogerian therapy, MI counselors use reflective listening to express understanding of the client's feelings and experience, and considerable effort is placed on understanding the client's subjective reality. Although both MI and Rogerian therapy accept that change is ultimately up to the client, MI can be more directive and goal oriented (Rollnick \& Miller, 1995). For example, when MI is applied in health promotion and public health settings, there may be desired outcomes for clients to modify behavior in a specific direction (e.g., quitting smoking or decreasing fat intake). Whereas in Rogerian psychotherapy a goal may be to help the client accept and integrate incongruent behaviors or socially unacceptable attributes, in MI greater emphasis is placed on resolving these discrepancies and building motivation for change (Patterson, 1986).

Although apparently MI is similar to the Trans theoretical model (TTM), in that both models emphasize the need to match interventions to the client's readiness and pros and cons for changing behavior and MI intervention protocols often incorporate some elements of "staging," there are subtle differences. 


\section{Motivational Interviewing Tool for Health Recovery}

The distinction between MI and TTM is in part due to pragmatics, in that MI is generally provided as a "real-time," face-to-face (or telephone) intervention, whereas most TTM interventions have been delivered through audiovisual modalities, without direct or immediate interpersonal interaction.

Motivational interviewing can also be distinguished from cognitive behavior therapy (CBT). CBT often involves the counselor confronting a client's irrational or maladaptive beliefs. MI, on the other hand, rarely involves direct confrontation of beliefs on the part of the counselor .However, the MI counselor may use reflective listening to clarify such beliefs and to "softly confront" how they influence current behaviors, as well as how these beliefs and actions may affect clients' ability to achieve happiness and their broader life goals.

\section{Efficacy of Motivational Interviewing in diverse health areas:}

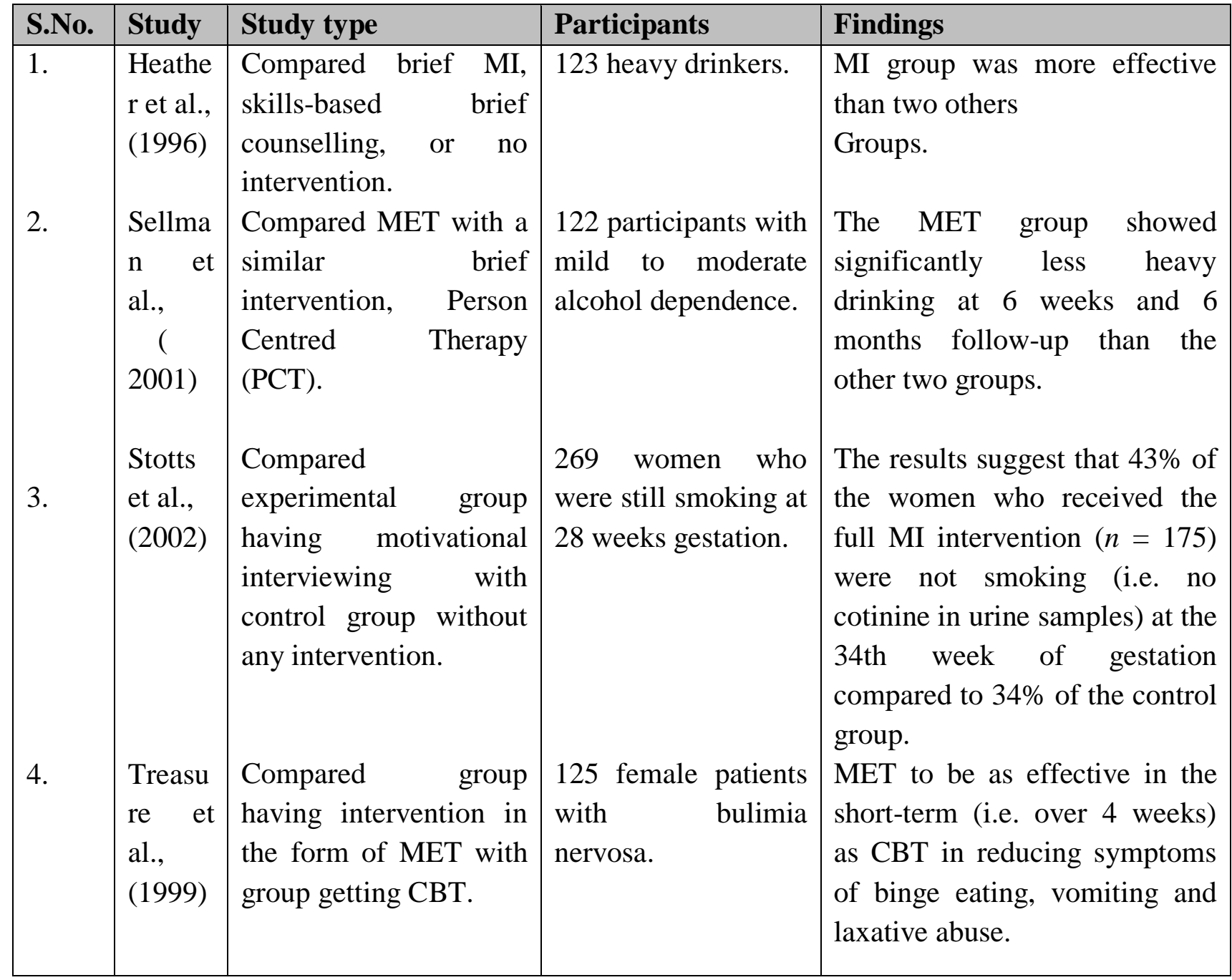




\section{CONCLUSION}

Motivational interviewing perceives to have broad application in health recovery and promotion. It provides practitioners with an effective means of working with patients who are ambivalent about, or not ready for change. Motivational interviewing is a key to unlock the door of a treasure in the form of human potential of which individual is not acquainted and is buried inside the crust due to ambivalence nature to change behavior. Motivational interviewing used to work as a traffic signal and gave direction on an unclear path. Questions remain, however regarding how MI works with different conditions and individuals and the impact of MI across different ethnic, age, and socio demographic populations.

Despite MI contribution in health behavior change, there are few controlled studies evaluating the efficacy of MI with heath. More research is needed to be in this direction.

\section{Acknowledgments}

The author appreciates all those who participated in the study and helped to facilitate the research process.

\section{Conflict of Interests}

The author declared no conflict of interests.

\section{REFRENCES}

Carkhuff, R. (1993). The art of helping (7th ed.). Amherst, MA: Human Resource Development Press

Dunn, C., Deroo, L., \& Rivara, F. P. (2001). The use of brief interventions adapted from motivational interviewing across behavioral domains: a systematic review. Addiction, 96(12), 1725-1742.

Emmons, K. M., \& Rollnick, S. (2001). Motivational interviewing in health care settings: opportunities and limitations. American journal of preventive medicine, 20(1), 68-74.

Heather, N., Rollnick, S., Bell, A., \& Richmond, R. (1996). Effects of brief counselling among male heavy drinkers identified on general hospital wards. Drug and alcohol review, 15(1), 29-38.

Miller WR, Rollnick SR. Motivational interviewing: preparing people to change behaviour. New York: Guilford Press; 1991.

Miller, W. R., \& Sanchez, V. C. (1994). Motivating young adults for treatment and lifestyle change.

Miller, W., \& Rollnick, S. (2002). Motivational interviewing: Preparing people for change. 2nd The Guilford Press. New York.

Noonan, W. C., \& Moyers, T. B. (1997). Motivational interviewing. Journal of Substance Misuse, 2(1), 8-16. 


\section{Motivational Interviewing Tool for Health Recovery}

Patterson, C. (1986). Theories of counseling and psychotherapy (4th ed.). New York: Harper \& Row.

Resnicow, K., DiIorio, C., Soet, J. E., Borrelli, B., Hecht, J., \& Ernst, D. (2002). Motivational interviewing in health promotion: it sounds like something is changing. Health Psychology, 21(5), 444.

Rollnick, S., \& Miller, W. R. (1995). What is motivational interviewing?.Behavioural and ncognitive Psychotherapy, 23(04), 325-334.

Sellman, J. D., Sullivan, P. F., Dore, G. M., Adamson, S. J., \& MacEwan, I. (2001). A randomized controlled trial of motivational enhancement therapy (MET) for mild to moderate alcohol dependence. Journal of Studies on Alcohol, 62(3), 389-396.

Stotts, A. L., DiClemente, C. C., \& Dolan-Mullen, P. (2002). One-to-one: a motivational intervention for resistant pregnant smokers. Addictive behaviors, 27(2), 275-292.

Treasure, J. L., Katzman, M., Schmidt, U., Troop, N., Todd, G., \& de Silva, P. (1999). Engagement and outcome in the treatment of bulimia nervosa: First phase of a sequential design comparing motivation enhancement therapy and cognitive behavioural therapy. Behaviour research and therapy,37(5), 405-418.

How to cite this article: S Kumar, M Srivastava, A Kumar, A Gupta (2016), Motivational Interviewing Tool for Health Recovery, International Journal of Indian Psychology, Volume 4, Issue 1, No. 69, ISSN:2348-5396 (e), ISSN:2349-3429 (p), DIP:18.01.007/20160401, ISBN:978$1-365-45447-9$ 